\title{
Eine neue Methode für die Bestimmung des Mikroplastik- Massenanteils in Umweltproben
}

\author{
Stefan Spacek · Ole Mallow · Therese Schwarzböck · Johann Fellner · Helmut Rechberger
}

Online publiziert: 27. Juli 2020

(C) Der/die Autor(en) 2020

\begin{abstract}
Zusammenfassung Die zunehmende Verschmutzung von aquatischen und terrestrischen Ökosystemen mit Mikroplastik stellt ein ernstzunehmendes Umweltproblem dar, wobei insbesondere die hohe Widerstandfähigkeit von Kunststoffen gegenüber Abbauprozessen zu einer stetigen Anreicherung von Mikroplastik in der Umwelt führt. Für die Bewertung der bereits vorhandenen Mikroplastikbelastung in der Umwelt

tate untermauern einerseits die hohe Wiederfindungsrate von Kunststoffen bei gespikten Mikroplastikproben (im Bereich von 93 bis $110 \%$ ) und andererseits eine ungefähre Nachweisgrenze der Methode, die im Bereich von 1 Massen-\% Mikroplastikgehalt liegt. Die EAOEM positioniert sich als verhältnismäßig einfaches und kostengünstiges Messverfahren, um Mikroplastik in Umweltproben zu bestimmen.
\end{abstract} sowie der Evaluierung von Haupteintragsquellen ist die Entwicklung geeigneter Methoden für die akkurate und einfache Bestimmung von Mikroplastik ein entscheidender Faktor. Die im gegenständlichen Artikel vorgestellte Analysenmethode stellt eine derartige Möglichkeit für die massenbezogene Analyse von Mikroplastik dar. Die an der TU Wien entwickelte EA-OEM (Elemental Analysis Overdetermined Equation Method) kann als thermoanalytische Methode eingestuft werden und nutzt die ausgeprägten Unterschiede in der Elementarzusammensetzung zwischen Kunststoffen, biogenen und anorganischen Materialien. Daraus wird der Gehalt an Mikroplastik in Umweltproben quantitativ bestimmt. Die EA-OEM wurde in den letzten Jahren anhand verschiedener Probentypen getestet und durch zusätzliche Wiederfindungsversuche mit gespikten Matrixproben und der Teilnahme an einem Ringversuch bereits einer eingehenden Validierung unterzogen. Die gegenständliche Arbeit fasst einige dieser Untersuchungen zusammen. Diese Resul-

DI S. Spacek, Bakk.techn. (西) ·

Dr. O. Mallow, MSc .

Dr. T. Schwarzböck, MSc .

Assoc. Prof. DI Dr. J. Fellner .

Univ.-Prof. DI Dr. H. Rechberger

Institut für Wassergüte und

Ressourcenmanagement, Forschungsbereich Abfallwirtschaft und Ressourcenmanagement, Technische Universität Wien, Karlsplatz 13, 1040 Wien, Österreich stefan.spacek@tuwien.ac.at
Schlüsselwörter Mikroplastik · EAOEM - Thermoanalytische Verfahren . Monitoring

\section{A new method for determining microplastic content in environmental samples}

Abstract The increasing pollution of aquatic and terrestrial ecosystems with microplastics is a severe problem for the environment. Especially the high resistance of plastics to degradation processes causes a constant accumulation of microplastics in the environment. The development of suitable methods for the accurate and simple determination of microplastics is a vital factor to evaluate existing microplastic accumulations in the environment. The method presented here describes a thermoanalytical method for massbased microplastic determination. The EA-OEM (Elemental Analysis Overdetermined Equation Method) was developed at TU Wien and makes use of the distinct differences in the elemental composition of plastic, biogenic and inorganic materials to quantitatively measure the content of microplastics in environmental samples. The EA-OEM has been optimized in past years using polymer spiked samples and additionally evaluated in an inter-laboratory round robin test. The aim of this paper is to summarize recent investigations. The results demonstrate good recovery rates for microplastics in polymer spiked samples (in the range of 93 to $110 \%)$. Furthermore, the limit of de- tection was estimated with $1 \mathrm{wt} \% \mathrm{mi}-$ croplastics. The EA-OEM poses a comparatively simple and cost-effective method to determine microplastics in environmental samples.

Keywords Microplastics · EA-OEM • Thermoanalytical Methods .

Monitoring

\section{Einleitung}

Die Verschmutzung von aquatischen und terrestrischen Ökosystemen durch den kontinuierlichen Eintrag von Kunststoffabfällen sowie Mikroplastik (MP) stellt unsere Gesellschaft aktuell vor große Herausforderungen (Derraik 2002; Law und Thompson 2014). Der möglichst akkuraten Quantifizierung von Mikroplastik-Eintragsquellen sowie der Bestimmung von bereits bestehenden Hintergrundbelastungen in der Umwelt wird dabei eine wichtige Rolle zuteil. Dies kann nur durch fortlaufende Bestrebungen in Richtung Entwicklung und Optimierung von geeigneten Analysenmethoden sowie der Standardisierung und Normung dieser Methoden erreicht werden. Methodisch betrachtet, kann der Großteil der sich derzeit in Entwicklung befindlichen Analysenmethoden in zwei Hauptgruppen eingeteilt werden (La Nasa et al. 2020): i) Mikroskopische \& spektroskopische Methoden und ii) Thermoanalytische Methoden. Innerhalb der ersten Hauptgruppe können MP-Analysen vielfach bereits mit einfachen Lichtmikroskopen durchgeführt werden. Eine eindeutige optische Differenzierung zwischen Partikeln biogenen Ursprungs und Plastiktfragmenten ist in diesem Fall jedoch nicht immer gewährleistet. Aus diesem Grund hat sich in den letzten Jahren die Kopplung von Mikroskopiertechniken mit spektroskopischen Detektionsmethoden etabliert (Prata et al. 2019). Mithilfe von Infrarotspektroskopie (FTIR) oder Raman-unterstützten mikroskopischen Untersuchungen, kann das FTIRoder Raman-Spektrum jedes einzel- 
nen Partikels aufgenommen werden und dadurch die Materialzusammensetzung in den meisten Fällen eindeutig bestimmt werden. Um die Präzision der Zuordnung von Partikeln noch weiter $\mathrm{zu}$ erhöhen, gehen aktuelle methodische Weiterentwicklungen dieses Ansatzes in Richtung Kombination von FTIR- und Raman-Spektren (Brandt et al. 2020). Bildgebende Analysen liefern in erster Linie Informationen $\mathrm{zu}$ Partikelanzahl, Größenverteilung, vorhandenen Partikelformen sowie Polymertypen. Diese Parameter sind wichtig für die Beantwortung ökotoxikologischer Fragestellungen. Die Partikelanzahl kann zudem als Indikator für die Grundbelastung von MP in der Umwelt herangezogen werden. Eine umfassende und vergleichbare Charakterisierung der MP-Belastung ist jedoch nur mit der Bestimmung von massebezogenen Parametern möglich, da nur damit der mengenmäßige Eintrag von MP in die Umwelt beschrieben werden kann. Die Umrechnung von der Partikelanzahl und Partikelgröße auf die Gesamtmasse ist zwar möglich, jedoch sind diese Berechnungen häufig mit sehr hohen Unsicherheiten verbunden. Um zukünftige Analysenergebnisse zu MPBelastungen eindeutig vergleichbar $\mathrm{zu}$ machen, bedarf es daher zusätzlicher Methoden, welche den Massenanteil von MP in Umweltproben analysieren können.

Der Großteil der zurzeit in der Literatur beschriebenen massebezogenen Methoden gehört der zweiten Hauptgruppe an. Diese wird derzeit am besten unter dem Begriff thermoanalytische Methoden zusammengefasst. Thermoanalytische Methoden basieren auf der Verbrennung oder Pyrolyse des Probenmaterials und anschließender Auftrennung, Detektion sowie Quantifizierung der dabei entstehenden Gase (La Nasa et al. 2020). Diese Gase, beziehungsweise deren Verhältnisse zueinander, sind jeweils stoffspezifisch und ermöglichen dadurch einen Rückschluss auf die ursprüngliche Zusammensetzung der Feststoffprobe. Dies ermöglicht die Bestimmung von Kunststoffanteilen und zumeist auch die Detektion der Kunststofftypen. Im Gegensatz zu mikroskopischen Untersuchungen besteht bei thermoanalytischen Methoden keine Einschränkung bezüglich der Größe der erfassten Partikel, da durch die vollständige Verbrennung oder Pyrolyse der Gesamtkunststoffgehalt einer Probe erfasst wird. Als
Hauptvertreter, bezogen auf die Veröffentlichungen in den vergangenen Jahren, sind die Methoden TED-GCMS (Thermoextraktion DesorptionGaschromatographie-Massenspektrometrie, Dümichen et al. 2017) und Pyrolyse-GC-MS (Pyrolyse-Gaschromatographie-Massenspektrometrie, Fischer und Scholz-Bottcher 2017) zu nennen.

Eine weitere Methode, um MP massebezogen zu bestimmen, wurde jüngst entwickelt und ist Gegenstand dieses Artikels. Die sogenannte Elemental Analysis combined with Overdetermined Equation Method (EA-OEM) basiert in erster Linie auf der Analyse der makro-elementaren Zusammensetzung einer Probe (CHNSO-Bestimmung). Die EA-OEM wird daher thematisch zu den thermoanalytischen Methoden gezählt. Sie erlaubt eine kostengünstige sowie im Routinebetrieb einfach durchzuführende Bestimmung des massebezogenen Summenparameters „Gesamtkunststoffanteil“ für die MP-Belastung in Umweltproben. Details zu der Methodik und erste Ergebnisse wurden von Mallow et al. (2020) veröffentlicht. Ziel des gegenständlichen Artikels ist es, weiterführende Ergebnisse zu Untersuchungen und Weiterentwicklung der EA-OEM darzulegen und damit die Anwendbarkeit und auch Einschränkungen der Methodik zu diskutieren. Insbesondere werden Untersuchungen zu Messbereich, Einfluss von Matrixeffekten und der Nachweisgrenze beschrieben.

\section{EA-OEM und die adaptierte Bilanzenmethode}

Das Messprinzip der EA-OEM für die Bestimmung von MP basiert grundsätzlich auf der adaptierten Bilanzenmethode (aBM), eine an der TU Wien am Institut für Wassergüte und Ressourcenmanagement entwickelte Methode für die Bestimmung des Kunststoffanteils in Ersatzbrennstoffen (Fellner et al. 2011; Schwarzböck et al. 2018). Die Methode wurde in den vergangenen Jahren konsequent weiterentwickelt und auf die Quantifizierung von Mikroplastik in Umweltproben angepasst. Die Bestimmung des unbekannten MP-Anteils erfolgt dabei durch die Unterscheidung von Kunststoffen und biogenen Materialien anhand deren unterschiedlicher makro-elementarer Zusammensetzung (Gehalt an Kohlenstoff C, Wasserstoff H, Stickstoff N, Schwefel S, Sauerstoff O). So bestehen biogene Materialen $\mathrm{zu}$ - meist $\mathrm{zu} 40$ bis $50 \%$ aus Sauerstoff. Kunststoffe hingegen enthalten entweder keinen Sauerstoff (PE, PP, PVC, PS) oder einen geringeren Anteil (PUR, PET). Der Kohlenstoffgehalt von Kunststoffen ist deutlich höher als von biogenen Materialien (PE/PP: $86 \%$, PS: $92 \%$, PET: $65 \%$ ). Als Ausnahme muss an dieser Stelle die Stoffklasse der Fette genannt werden (Abb. 1). Diese weist eine CHNSO-Zusammensetzung auf, welche näher an der Gruppe der Kunststoffe liegt. Da Fette in durchaus höheren Mengen in Umweltproben vorhanden sein können, müssen diese im Zuge der Probenaufbereitung für die EAOEM durch Extraktion oder Verseifung der Probe vollständig entfernt werden, damit sie nicht fälschlicherweise als Kunststoffe detektiert werden.

\subsection{Instrumentelle Elementaranalyse (CHNSO-Bestimmung)}

Für die Anwendung der EA-OEM ist die Bestimmung der makro-elementaren Zusammensetzung (CHNSO-Zusammensetzung) in der Probe nötig. Durch die unterschiedliche CHNSO-Zusammensetzung von Kunststoffen und biogenen Materialien ermöglicht diese Bestimmung eine quantitative Aussage über den MP-Anteil in Umweltproben. Die Messung der Elemente C, $\mathrm{H}$, $\mathrm{N}$, und S basiert im Grunde auf der vollständigen Oxidierung der $\mathrm{zu}$ analysierenden Probe bei hohen Verbrennungstemperaturen von über $1000^{\circ} \mathrm{C}$. Die entstehenden Verbrennungsgase werden durch spezifische Adsorptionssowie Desorptionsvorgänge voneinander separiert. Durch die anschließende Messung der Gaskonzentrationen kann auf die ursprüngliche CHNS-Zusammensetzung rückgeschlossen werden. Dieses Messprinzip ist automatisiert und im Routinebetrieb einsetzbar. Die Bestimmung von Sauerstoff verläuft ähnlich, jedoch wird die Probe unter Sauerstoffausschluss pyrolysiert anstatt oxidiert.

\subsection{Berechnung des MP Anteils}

Die aus der Elementaranalyse erhaltenen CHNSO-Messwerte $(T x)$ beziehen sich auf die trockene Gesamtprobe inklusive des Aschegehalts $(A)$. Um die für die Methode benötigten wasserund aschefreien TO $x$ ( $x=$ CHNSO)-Werte zu erhalten, müssen anschließend noch der Aschegehalt und die dazugehörige CHNSO-Zusammensetzung der 


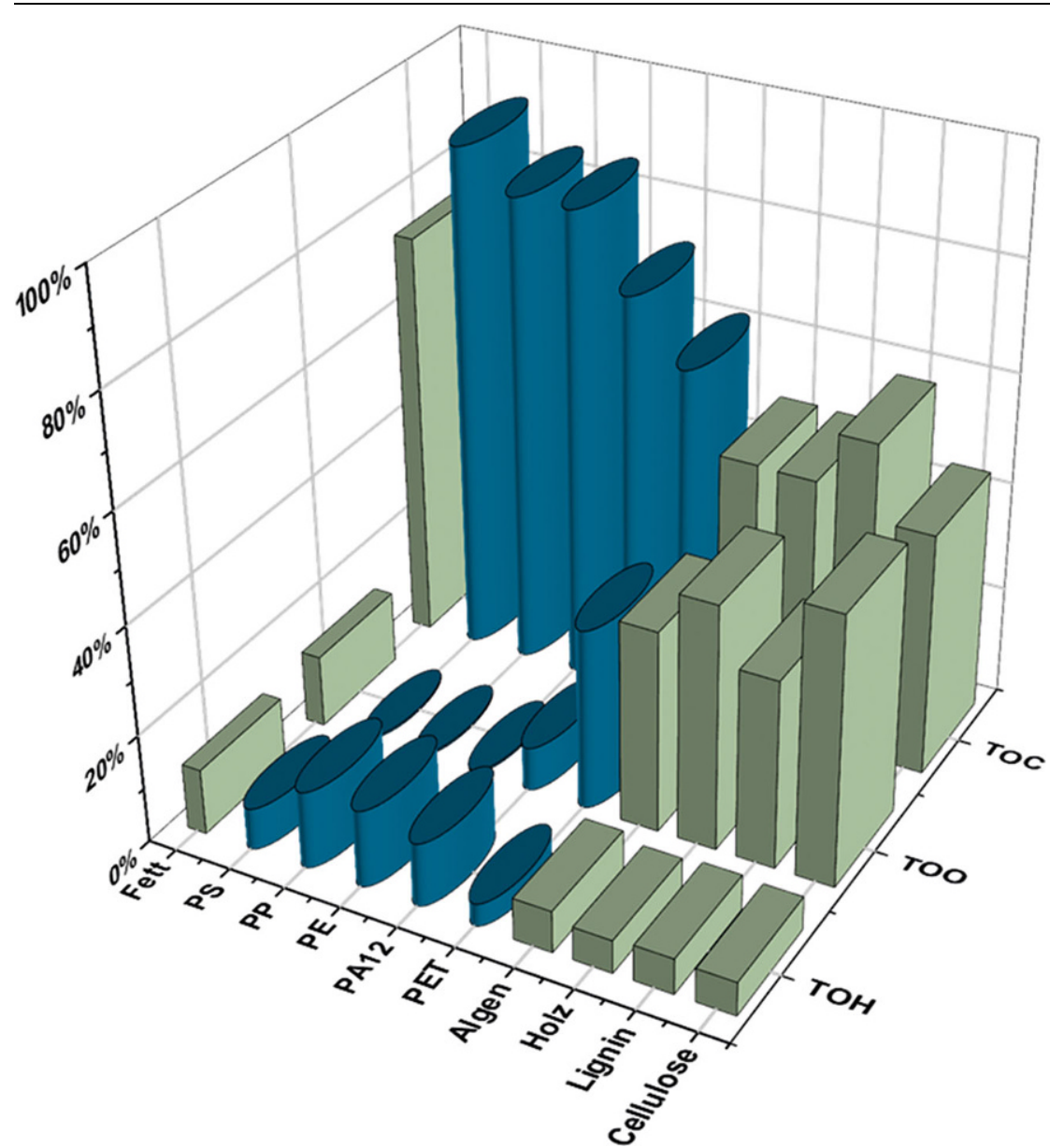

Abb. 1 Massenanteile an $\mathrm{C}, \mathrm{H}$, und $\mathrm{O}$ (auf wasser- und aschefreier Basis, angegeben als TOC, TOH und TOO) in Materialien, die in Umweltproben häufig vorkommen (Algen, Holz, Lignin, Cellulose, Fett) sowie eine Auswahl an Hauptkunststoffen, welche häufig bei Mikroplastikuntersuchungen detektiert werden. Die Werte für Algen, Fett und Holz basieren auf eigenen Messungen. Die Werte für die Polymertypen wurden anhand ihrer chemischen Summenformel berechnet. Die Angaben für Lignin beziehen sich auf extrahiertes Lignin aus einer Mischung von verschiedenen Harthölzern und stammen aus Jahan et al. (2012)

Asche $(T I x)$ bestimmt werden. Anhand Gl. 1 können im Anschluss daran die wasser- und aschefreien TOx-Gehalte berechnet werden.

$$
T O x=(T x-T I x \cdot A) /(1-A)
$$

Die $T O x$-Werte der Probe $\left(\right.$ TOx $\left._{\text {Probe }}\right)$ repräsentieren die chemische Zusammensetzung der in der Probe vorhandenen, vermischten biogenen Fraktion und Kunststofffraktion. Diese Zusammensetzung muss der TOx-Zusammensetzung der beiden Reinstofffraktionen biogen $\left(T O x_{\mathrm{B}}\right)$ und Kunststoff $\left(T O x_{\mathrm{K}}\right)$ multipliziert mit den dazugehörigen Massenanteilen der biogenen Fraktion $\left(x_{B}\right)$ und der Kunststofffraktion $\left(x_{K}\right)$ entsprechen (Gl. 2). Die Massenanteile $\left(x_{B}\right.$ und $\left.x_{K}\right)$ in dieser Bilanz sind je-
2.3 CHNSO-Zusammensetzung der Reinstofffraktionen

Die von den AutorInnen in den letzten Jahren gesammelten Erfahrungen zeigen, dass insbesondere die Bestimmung der CHNSO-Zusammensetzung der biogenen Reinfraktion sowie der Kunststoff-Reinfraktion $\left(T O x_{\mathrm{B}}\right.$ und $\left.T O x_{\mathrm{K}}\right)$ herausfordernd sein kann. Da diese Werte wichtige Parameter für das Analysemodell sind, sollten sie jedoch möglichst genau bestimmt werden. Anders als bei der Anwendung der aBM für Ersatzbrennstoffe, kann die CHNSO-Zusammensetzung der beiden Reinfraktionen nicht durch händisches Sortieren eindeutig abgeleitet werden. Für eine schnelle Erstabschätzung der Mikroplastikbelastung in Umweltproben ist die Elementaranalyse daher, aus derzeitiger Sicht, nur eingeschränkt geeignet. In den vergangenen Jahren durchgeführte Untersuchungen zeigen jedoch, dass die MP-Polymer-Zusammensetzung in den unterschiedlichen Umweltmatrizen häufig sehr ähnlich ist und zumeist von einem hohen Anteil an PE dominiert wird. Dadurch können für Erstabschätzungen des Mikroplastikgehalts Näherungswerte zur chemischen Zusammensetzung der vorhandenen Kunststoffe herangezogen werden. Abseits davon besteht für die Entwicklung des Analysemodells auch die Möglichkeit, dass für eine Erstbestimmung alternative thermoanalytische Methoden wie zum Beispiel TED-GC-MS oder mikroskopische Untersuchungen zum Einsatz kommen.

\subsection{Kurzbeschreibung der EA-OEM}

Die EA-OEM kann anhand der folgenden Punkte zusammengefasst werden:

- Unterteilung der Gesamtprobe in die 3 Fraktionen biogener Anteil, Kunststoffanteil sowie Mineralik- und Inertanteil, wobei die biogene Fraktion und die Kunststofffraktion eine signifikant unterschiedliche makroelementare Zusammensetzung aufweisen müssen.

- Bestimmung der makro-elementaren Zusammensetzung (CHNSO-Bestimmungen) der wasserfreien $\mathrm{Ge}$ samtprobe $(T x)$.

- Bestimmung des Aschegehalts aus der Gesamtprobe $(A)$ und anschließende Messung der dazugehörigen CHNSO-Zusammensetzung der Asche (TIx). 

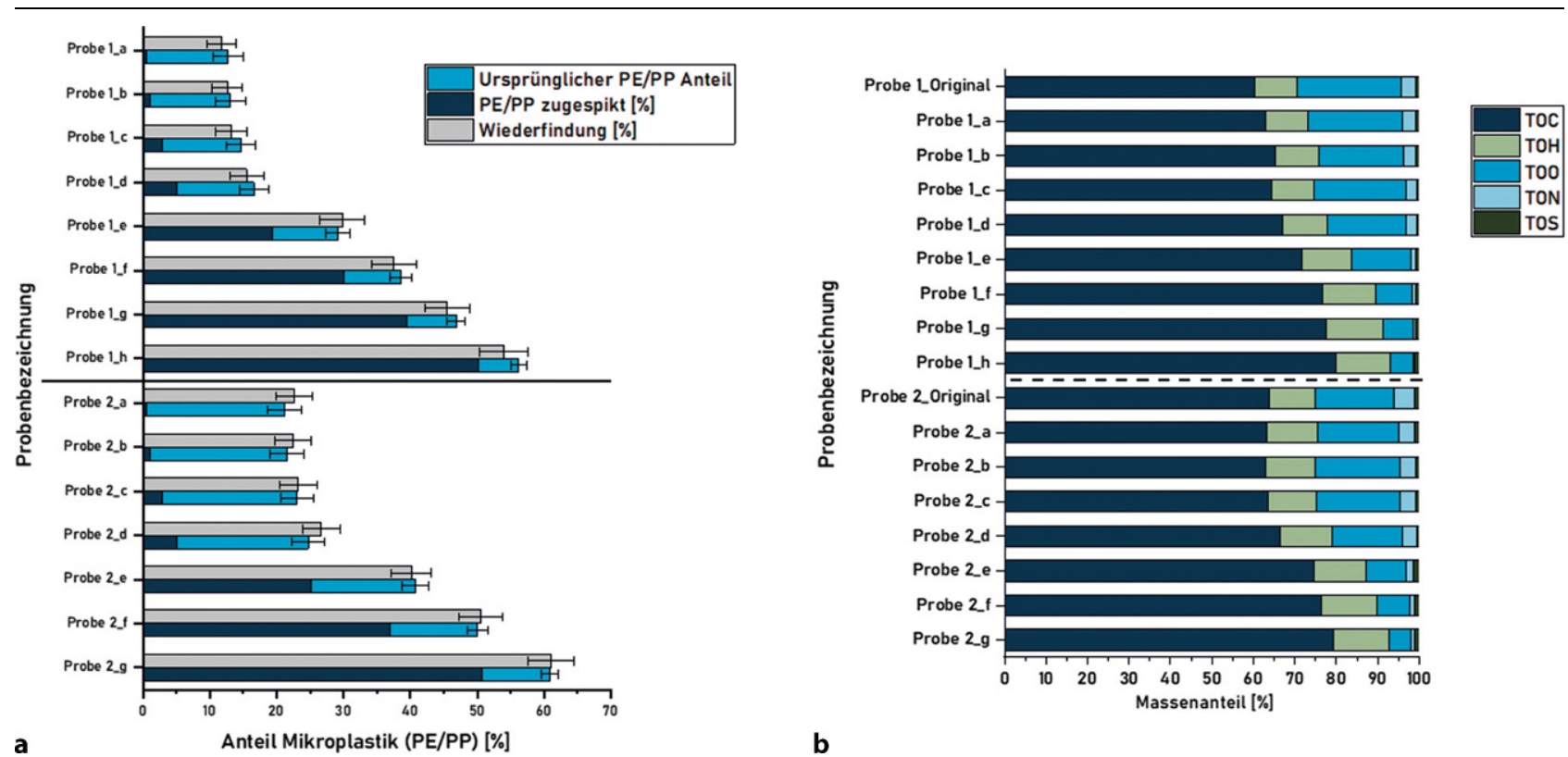

Abb. 2 Zusammenfassung der Ergebnisse des Wiederfindungsversuchs 1. Grafik a Gegenüberstellung der theoretischen PE/PPAnteile in matrixgespikten Proben und der anschließenden Wiederfindung (Mallow et al. 2020). Grafik b Veränderung der Verteilung derTO $x$ Werte in Abhängigkeitvon derdazugespikten PE/PP-Menge. DadieSummedergemessenenTO $x$-WerteaufgrundvonMessunsicherheiten nicht genau $100 \%$ ergibt, wurden die Ergebnisse für die Abbildung auf $100 \%$ normiert

- Berechnung der asche- und wasserfreien TO $x$-Werte $(x=$ CHNSO) anhand Gl. 1.

- Einmalige Bestimmung der probenspezifischen TOx-Werte der biogenen Reinstofffraktion und der Kunststoffreinfraktion.

- Erstellung eines Gleichungssystems, bei dem die bestimmten TOx-Werte einer Probe den TOx-Werten der Reinfraktionen, multipliziert mit den jeweiligen Massenanteilen, gegenübergestellt werden (Gl. 2).

- Berechnung der beiden unbekannten Massenanteile von biogener Fraktion und Kunststofffraktion mithilfe einer nichtlinearen Ausgleichsrechnung.

\section{Ergebnisse}

\subsection{Wiederfindungsversuch 1}

Aufgrund des derzeitigen Mangels an für die MP-Analytik geeigneten Referenzmaterialien, wurde die Analysenperformance der EA-OEM anhand von Wiederfindungsversuchen überprüft. Für den Wiederfindungsversuch 1 wurden zwei verschiedene Feststoffproben herangezogen, die aus Abwasserproben eines Kunststoffherstellers abgetrennt wurden. Diese Feststoffproben enthielten bereits Mikroplastik in Form von PE + PP und wurden mit zusätzlichem
PE/PP (im Verhältnis 1:1, Korngröße $<500 \mu \mathrm{m})$ in aufsteigenden Konzentrationen von 0,5 bis $50 \%$ MP gespikt. Anhand der Analysenergebnisse sowie der Wiederfindungsrate wurden einerseits der Messbereich der Methode überprüft und andererseits das Auftreten von Matrixeffekten bei der Analyse bestimmt. Abb. 2 zeigt die Ergebnisse des Versuchs 1. Die Wiederfindungsraten liegen zwischen 93 und $110 \%$ und liefern damit eine erste Bestätigung für die Anwendbarkeit der EA-OEM zur Quantifizierung des Gehalts an MP.

\subsection{Wiederfindungsversuch 2}

Da die beim Wiederfindungsversuch 1 verwendeten Feststoffproben bereits eine Grundkontamination mit MP aufwiesen, konnte anhand der zuvor beschriebenen Ergebnisse keine absolute Nachweisgrenze für die EA-OEM bestimmt werden. Im Zuge von weiterführenden Versuchen wurde dies daher nachgeholt. Für den Wiederfindungsversuch 2 wurde Probenmaterial (biogene Probenmatrix), welches möglichst stark von den TO $x$-Werten von PE/PP abweicht, ausgewählt und mit PE/PP gemischt. Der zweite Versuch beschreibt daher die Nachweisgrenze der EA-OEM in einem optimalen Szenario. Es wurde ein im Zuge des Projekts BASEMAN WP2 (JPI Oceans) zur Verfügung gestelltes Probenmaterial herangezogen. Die Probe bestand aus gezüchteten, zerkleinerten Miesmuscheln und wurde vor dem Versand mit Wasser vermengt. Die Probe wurde im Labor gefriergetrocknet, homogenisiert und anschließend mit einer PE/PPMischung (Verhältnis 1:1, Korngröße $<500 \mu \mathrm{m})$ in den Konzentrationen $1 \%$, $3 \%$ sowie $5 \%$ gespikt. Abb. 3 zeigt die erhaltenen Wiederfindungsraten der drei mittels EA-OEM gemessen Proben. Unter den oben beschriebenen optimalen Bedingungen, d.h. die TOx-Werte der biogenen Probenmatrix als auch die TO $x$-Werte für die Kunststofffraktion sind mit hoher Genauigkeit (geringer Unsicherheit) bekannt, konnten sehr gute Wiederfindungsraten für den Bereich 1 bis $5 \%$ MP erzielt werden. Eine Nachweisgrenze von etwas über $1 \%$ konnte anhand dieser Ergebnisse bestimmt werden. Veränderte Bedingungen können jedoch die Nachweisgrenze nach oben hin verschieben. Dies ist vorwiegend davon abhängig, wie akkurat die TO $x$-Werte der beiden Reinstofffraktionen bekannt sind bzw. bestimmt werden können. Zusätzlich ist auch die Unterschiedlichkeit der makro-elementaren Zusammensetzung zwischen der biogenen Matrix und den enthaltenen Kunststoffen entscheidend. Je unterschiedlicher die CHNSO-Gehalte ausfallen, desto genauer sind auch 


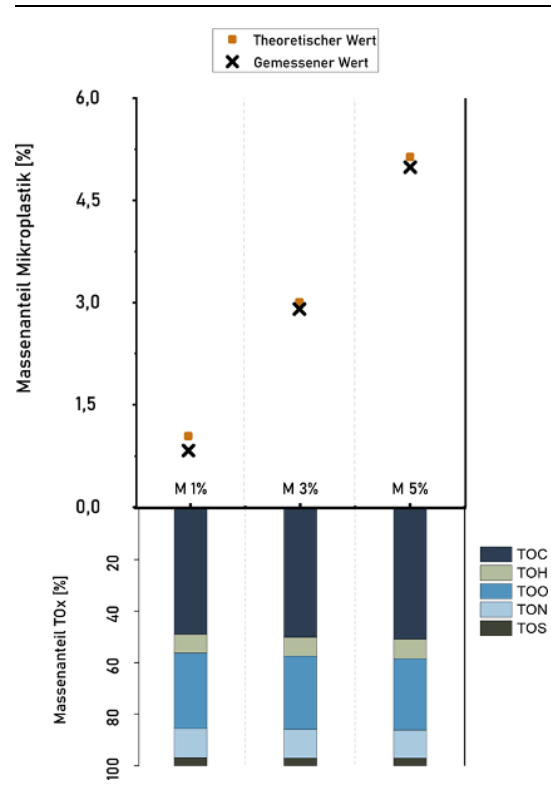

Abb. 3 Ergebnisse des Wiederfindungsversuchs 2: Der obere Teil der Grafik zeigt den theoretischen Wert sowie den durch EA-OEM ermittelten MP-Anteil von mit PE/PP gespiktem biogenen Probenmaterial. Die verhältnismäßig geringen MPAnteile wurden deshalb gewählt, um eine initiale Abschätzung der Nachweisgrenze für die EA-OEM zu erhalten. Die unteren Balkendiagramme zeigen die Verteilung der TO $x$-Werte der drei gespikten Proben. DadieSumme dergemessenen TO $x$ Werte aufgrund von Messunsicherheiten nicht genau $100 \%$ ergibt, wurden die Ergebnisse für die Abbildung auf $100 \%$ normiert

die Ergebnisse der EA-OEM. Die Analysengenauigkeit beeinflusst in weiterer Folge auch die Nachweisgrenze. Eine allgemeingültige Aussage über die Sensitivität der Methode lässt sich daher zum aktuellen Zeitpunkt nicht treffen. Sicher ist jedoch, dass diese von den jeweiligen Spezifikationen des Probenmaterials abhängt. Die Abhängigkeit der Bestimmungsgenauigkeit des MPGehalts vom enthaltenen Aschegehalt kann vom derzeitigen Erfahrungsstand aus als gering beurteilt werden.

\subsection{Ringversuch}

Weitere Erkenntnisse zur Analysenperformance der EA-OEM konnten im $\mathrm{Zu}$ ge eines Ringversuchs gewonnen werden. Dieser wurde von der deutschen Bundesanstalt für Materialforschung und -prüfung (BAM) durchgeführt. Für diese Studie wurde eine synthetische Umweltprobe mit definiertem, aber für die Teilnehmer unbekanntem Analytgehalt an 11 verschiedene Testlabors versendet (Becker et al. 2020). Ziel war es, erste Erfahrungen über die allgemeine Eignung von thermoanalytischen Methoden hinsichtlich der Bestimmung des MP-Gehalts zu sammeln. Die Probe bestand aus einer kunststofffreien Matrix und wurde mit den vier Polymeren PE, PP, PS und PET gespikt. Die vier eingesetzten Polymertypen waren bekannt, ihre jeweiligen Anteile zueinander jedoch nicht. Zusätzlich wurde die kunststofffreie Matrix für den Ringversuch zur Verfügung gestellt. Vor Beginn der Analysen für den Ringversuch wurden intern folgende Forschungsfragen formuliert:

- Ist es möglich, die unbekannte TOxZusammensetzung der reinen Kunststofffraktion in der Probe, die einen essenziellen Eingangsparameter für die EA-OEM darstellt, anhand alternativer Strategien abzuschätzen?

- Standardmäßig wird mittels EA-OEM nur der Summenparameter MP erfasst. Lassen die gemessenen TO $x$ Werte der Kunststoffreinfraktion Rückschlüsse auf die Massenverteilung von PE/PP, PS und PET zu?

Abb. 4 zeigt das Ergebnis der mittels der EA-OEM durchgeführten Bestimmungen. Der Sollwert von $45,9 \mu \mathrm{g} / \mathrm{mg}$ Gesamtkunststoff in der zur Verfügung gestellten Probe wurde mit dem gemessenen Wert von $44,7 \mu \mathrm{g} / \mathrm{mg}$ sehr gut wiedergefunden. Der Gesamtkunststoffgehalt entspricht einem Anteil von $4,5 \%$ in der Gesamtprobe und liegt somit über der zuvor beschriebenen, theoretischen Nachweisgrenze der Me- thodik. Vor der Bestimmung des Gesamtkunststoffgehalts wurde die unbekannte Verteilung der eingesetzten Kunststoffe (Anteile an PE, PP, PS und PET) anhand einer Extraktion des vorhandenen Gesamtkunststoffs aus der gespikten Matrixprobe ermittelt. Hierfür wurde eine Dichteseparation mit gesättigtem $\mathrm{NaI}$ und eine anschließende oxidative Aufreinigung $\left(\mathrm{H}_{2} \mathrm{O}_{2}\right)$ durchgeführt, um den Kunststoffanteil möglichst vollständig von der restlichen Matrix abzutrennen. Von dieser Reinfraktion wurde anschließend die elementare Zusammensetzung bestimmt und als Inputparameter für $T O x_{\mathrm{K}}$ für Gl. 2 herangezogen. Die sehr gute Annäherung an den tatsächlichen Gesamtkunststoffgehalt zeigt dabei, dass die beschriebene Aufreinigung eine Möglichkeit darstellt um eine unbekannte Kunststoffzusammensetzung bestimmen zu können.

Da mittels EA-OEM standardmäßig nur der Gesamtkunststoffgehalt ermittelt wird und die Anteile der einzelnen Polymere nicht erfasst werden, wurde die Verteilung der Polymertypen in der Probe abgeschätzt. Dazu wurden die durch Extraktion bestimmten TOx $_{\mathrm{K}^{-}}$ Werte erneut in ein Gleichungssystem eingefügt (angelehnt an die Gl. 2, wobei $T O x_{\mathrm{K}}$ anstatt $T O x_{\text {Probe }}$ eingesetzt wurde und die Gleichungen um 3 Fraktionen erweitert wurden: PE/PP, PS und PET), mit dem Ziel, die Massenanteile der einzelnen Polymere in der Kunststoffmischung abzuschätzen. Die Berechnung ergab Anteile von $60,1 \%$ PE/PP, $0,3 \%$ PS und $39,6 \%$ PET. Diese Bestimmung der Polymeranteile in der Kunststoffmischung wurde jedoch nicht genauer überprüft und kann daher nur als grobe Abschätzung verstanden werden. Tab. 1 stellt die Sollwerte mit der ermittelten Kunststoffverteilung gegenüber. Die PE/PP-Anteile sowie die PET-Anteile konnten sehr gut bestimmt werden (Wiederfindungsraten von 104 und 99\%). Einzig der PS-Anteil konnte nicht korrekt bestimmt werden (Wiederfindung bei nur knapp 6\%). Ein

Tab. 1 Ermittelte Wiederfindungsraten des Ringversuchs. Zusätzlich zu der Bestimmung des Summenparameters „Gesamtkunststoff" wurde im Zuge des Ringversuchs erstmals auch die Verteilung der Kunststoffe abgeschätzt. Diese Modellrechnung anhand der TO $x$-Werte der Kunststoffreinfraktion ergab sehr gute Wiederfindungsraten für PE/PP und PET. PS wurde in dem Modell zu gering eingeschätzt und vermutlich eher der PE/PP-Fraktion zugeordnet

\begin{tabular}{|c|c|c|c|}
\hline Polymer & Theoretischer Wert $[\mu \mathrm{g} / \mathrm{mg}]$ & Gemessener Wert [ug/mg] & Wiederfindungsrate [\%] \\
\hline Gesamtkunststoff & 45,9 & 44,72 & 97,4 \\
\hline $\mathrm{PE}+\mathrm{PP}$ & 25,7 & 26,74 & 104,0 \\
\hline PS & 2,2 & 0,13 & 5,9 \\
\hline PET & 18,0 & 17,85 & 99,2 \\
\hline
\end{tabular}



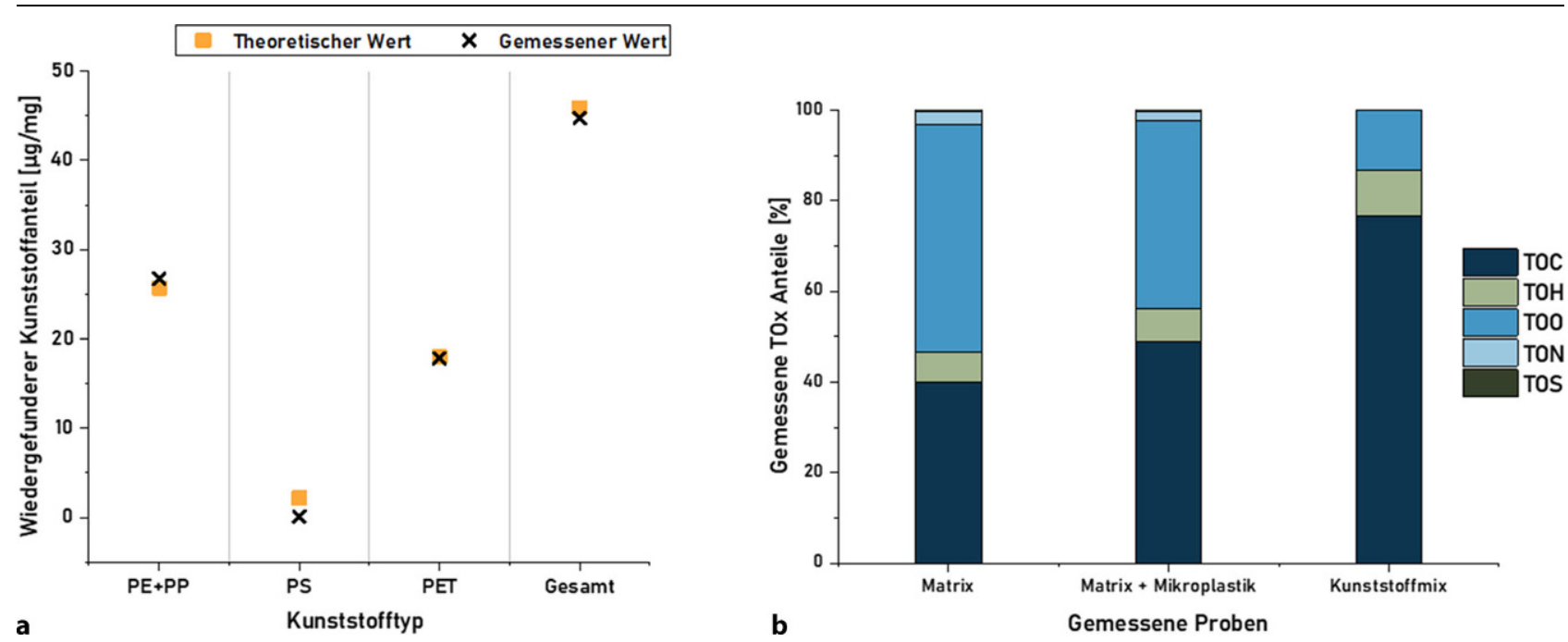

Abb. 4 Ergebnisse der EA-OEM-Messungen für die Proben des von der deutschen Bundesanstalt für Materialforschung und prüfung (BAM) organisierten Ringversuchs (Becker etal. 2020). Grafik a Gegenüberstellung der theoretischen Werte und der mittleren EA-OEM wiedergefundenen Kunststoffanteile. Grafik b TO $x$-Zusammensetzung ( $\mathrm{x}=\mathrm{CHNSO}$ ) der MP-freien Matrix, der unbekannten Testprobe (Matrix + Mikroplastik) und der im Labor durch Dichteseparation und Aufreinigung gewonnenen Kunststoffmischung aus der Testprobe (Kunststoffmix). Diese dargestellten Werte wurden für die Berechnung des MP-Anteils in der Probe mittels EA-OEM herangezogen

möglicher Grund hierfür ist die vergleichsweise niedrige Konzentration an PS in der Kunststoffmischung und die doch recht ähnliche makro-elementare Zusammensetzung von PS und PE/PP. Dadurch werden vermutlich PS-Anteile teilweise der PE/PP-Fraktion zugeordnet. Es kann davon ausgegangen werden, dass PS grundsätzlich im Zuge der EA-OEM mitbestimmt wird, da dieses Polymer im Verbrennungsofen genauso vollständig aufgeschlossen wird wie die anderen Polymere.

\section{Zusammenfassung und Ausblick}

Wie bereits Schwarzböck et al. (2016, 2018) in vorangegangenen Studien zur adaptierten Bilanzenmethode demonstrieren konnten, lässt sich der Kunststoffanteil in Abfallströmen sehr gut mithilfe der Elementaranalyse bestimmen. Die Anpassung der Methode für die MP-Bestimmung in Umweltproben war dabei methodisch gesehen nur noch ein kleiner Schritt. Hauptsächlich die deutlich geringeren Probemengen (oftmals weniger als $1 \mathrm{~g}$ Gesamtprobe) machten einige Anpassungen in der praktischen Durchführung der EA-OEM im Vergleich zur Anwendung auf Abfallproben notwendig. Die Ergebnisse der Wiederfindungsversuche sowie des Ringversuchs bestätigen dabei die $\mathrm{Zu}$ verlässigkeit der Methodik über einen weiten Messbereich.
Die Elementaranalyse (CHNSO-Bestimmung) als Kern der Methode stellt eine kosteneffiziente und mit verhältnismäßig geringem Aufwand $\mathrm{zu}$ betreibende Analysemethode dar, welche auch in kleineren Betriebslaboren für Routineuntersuchungen etabliert werden kann. Der gesamte organische Kohlenstoff (TOC Wert), als Summenparameter, ist beispielsweise eine häufig analysierte Messgröße in der Umweltanalytik. Die Erweiterung von TOC auf TO $x$ ( $x=$ CHNSO) ist zwar mit zusätzlichem Aufwand verbunden, jedoch bleibt die Methode zeit- und kosteneffizient. Zusätzlich ist das instrumentelle Messprinzip bereits seit Jahrzehnten im Einsatz und hat sich als robuste Methode erwiesen, die mit einem verhältnismäßig geringen Aufwand und überschaubarer Einschulung betrieben werden kann.

Der kritischste Punkt in der aktuellen Entwicklung liegt in der Bestimmung der Zusammensetzung der Reinstofffraktionen (CHNSO-Zusammensetzung der Kunststoffe und der biogenen Fraktion in der Probe), da diese, anders als bei Ersatzbrennstoffen, nicht durch händisches Sortieren bestimmt werden können. Vielmehr muss bei MP-Umweltproben meist mit Dichteseparation, chemischer Vorbehandlung oder einer Kombination mit anderen analytischen Methoden (z. B. TED-GC-MS, PyrolyseGC-MS oder FTIR-Mikroskopie) gearbeitet werden, um der geringen Proben- menge und den kleinen Partikelgrößen gerecht zu werden. Der dadurch entstehende Mehraufwand ist jedoch nur einmalig zu Beginn der Entwicklung eines Messmodells für eine spezifische Probenzusammensetzung notwendig. Laufende bzw. wiederholende Messungen des MP-Gehalts an denselben Probenahmeorten werden dadurch in Bezug auf Zeit und Kosten nicht beeinflusst. Eine geeignete Erstabschätzung der CHNSO-Zusammensetzung der Reinstofffraktionen (biogen und Kunststoffe in der Probe) ist oftmalig schon aufgrund des bereits beschriebenen engen Wertebereiches der CHNSOZusammensetzung (auf wasser- und aschefreier Basis) von jeweils biogenen und Kunststoffmaterialien möglich. Ergebnisse vergangener Untersuchungen deuten darauf hin, dass oftmals nur wenige Hauptkunststoffsorten (primär PE, PP, PA, PET) den Großteil der Mikroplastikbelastung in Umweltproben ausmachen, was die Charakterisierung der Kunststoffreinfraktion erleichtert. Weiters kann mithilfe alternativer Methoden wie TED-GC-MS, Pyrolyse-GCMS aber auch mikroskopischer Verfahren eine Erstbestimmung der Probenzusammensetzung hinsichtlich Polymertypen durchgeführt werden. Diese Methoden sind deutlich aufwendiger im Betrieb und mit höheren Kosten in der Geräteanschaffung verbunden. Für die einmalige Bestimmung notwendiger Eingangsdaten für die EA-OEM ist 
die Kombination aus verschiedenen Analysenmethoden jedoch vielversprechend.

Ein weiterer wichtiger Schritt in Richtung einer möglichst genauen Bestimmung von MP in Umweltproben ist die Entwicklung von standardisierten Probenaufbereitungsprotokollen. Dieser Aspekt ist nicht nur besonders wichtig für die verhältnismäßig wenig sensitive EA-OEM (Nachweisgrenze von MP beträgt im Optimalfall rund $1 \%$ ), sondern auch für den Rest der in der Literatur beschriebenen mikroskopischen und auch thermoanalytischen Methoden. Eine standardisierte Probenaufbereitung ist ein wesentlicher Faktor für akkurate und vergleichbare Analysenergebnisse.

Für eine ganzheitliche Betrachtung der Problematik Analytik von Mikroplastik in der Umwelt wird in Zukunft die Kombination von thermoanalytischen Methoden und mikroskopischen Untersuchungen, welche sich ebenfalls laufend weiterentwickeln, unumgänglich sein, um sowohl ökotoxikologisch wichtige Parameter wie Partikelgrößen und Partikelformen zu bestimmen und andererseits den massebezogenen MPGehalt als vergleichenden Parameter zu erhalten. Die Bestimmung der MPMassen in der Umwelt ist eine Größe, die dabei für nationale und internationale Vergleiche und Auswertungen zwingend notwendig ist. Mittels der EA-OEM kann der MP-Gehalt in Umweltproben massenbezogen bestimmt werden. Durch die zusätzlich auch kostengünstige Anwendung und die vielversprechenden Ergebnisse positioniert sich die vorgestellte Methodik daher innerhalb der bisher vorhandenen Methoden als potenzielles Messverfahren für ein routinemäßiges Monitoring von Mikroplastik in Umweltkompartimenten (z. B. zur Grenzwertüberwachung)

Funding Open access funding provided by TU Wien (TUW).

Open Access Dieser Artikel wird unter der Creative Commons Namensnennung 4.0 International Lizenz veröffentlicht, welche die Nutzung, Vervielfältigung, Bearbeitung, Verbreitung und
Wiedergabe in jeglichem Medium und Format erlaubt, sofern Sie den/die ursprünglichen Autor(en) und die Quelle ordnungsgemäß nennen, einen Link zur Creative Commons Lizenz beifügen und angeben, ob Änderungen vorgenommen wurden.

Die in diesem Artikel enthaltenen Bilder und sonstiges Drittmaterial unterliegen ebenfalls der genannten Creative Commons Lizenz, sofern sich aus der Abbildungslegende nichts anderes ergibt. Sofern das betreffende Material nicht unter der genannten Creative Commons Lizenz steht und die betreffende Handlung nicht nach gesetzlichen Vorschriften erlaubt ist, ist für die oben aufgeführten Weiterverwendungen des Materials die Einwilligung des jeweiligen Rechteinhabers einzuholen.

Weitere Details zur Lizenz entnehmen Sie bitte der Lizenzinformation auf http://creativecommons.org/licenses/ by/4.0/deed.de.

\section{Literatur}

Becker, R., Altmann, K., Sommerfeld, T., Braun, U. (2020): Quantification of microplastics in a freshwater suspended organic matter using different thermoanalytical methods-outcome of an interlaboratory comparison. Journal of Analytical and Applied Pyrolysis 148:104829. https://doi.org/10.1016/j.jaap.2020.104829

Brandt, J., Bittrich, L., Fischer, F., Kanaki, E., Tagg, A., Lenz, R., Labrenz, M., Brandes, E., Fischer, D., Eichhorn, K.J. (2020): High-Throughput Analyses of Microplastic Samples Using Fourier Transform Infrared and Raman Spectrometry. Appl Spectrosc. https://doi.org/10.1177/ 0003702820932926

Derraik, J.G.B. (2002): The pollution of the marine environment by plastic debris: a review. Ma Pollut Bull 44 (9):842-852. Doi https://doi.org/ 10.1016/S0025-326x(02)00220-5

Dümichen, E., Eisentraut, P., Bannick, C.G. Barthel, A.K., Senz, R., Braun, U. (2017): Fast identification of microplastics in complex environmental samples by a thermal degradation method. Chemosphere 174:572-584. https:// doi.org/10.1016/j.chemosphere.2017.02.010

Fellner, J., Aschenbrenner, P., Cencic, O., Rechberger, H. (2011): Determination of the biogenic and fossil organic matter content of refuse-derived fuels based on elementary analyses. Fuel 90
(11):3164-3171. https://doi.org/10.1016/j.fuel. 2011.06.043

Fischer, M., Scholz-Bottcher, B.M. (2017): Simultaneous Trace Identification and Quantification of Common Types of Microplastics in Environmental Samples by Pyrolysis-Gas Chromatography-Mass Spectrometry. Environ Sci Technol 51 (9):5052-5060. https://doi.org/10. 1021/acs.est.6b06362

Jahan, M.S., Liu, Z., Wang, H., Saeed, A., Ni, Y. (2012): Isolation and Characterization of Lignin from Prehydrolysis Liquor of Kraft-Based Dissolving Pulp Production. Cell Chem Technol 46 (3-4):261-267

La Nasa, J., Biale, G., Fabbri, D., Modugno, F (2020): A review on challenges and developments of analytical pyrolysis and other thermoanalytical techniques for the quali-quantitative determination of microplastics. Journal of Analytical and Applied Pyrolysis 149:104841. https:// doi.org/10.1016/j.jaap.2020.10484

Law, K.L., Thompson, R.C. (2014): Microplastics in the seas. Science 345 (6193):144-145. https:/ / doi.org/10.1126/science.1254065

Mallow, O., Spacek, S., Schwarzböck, T, Fellner, J., Rechberger, H. (2020): A new thermoanalytical method for the quantification of micropla- stics in industrial wastewater. Environ Pollut 259. doi:ARTN 11386210.1016/j.envpol.2019.113862 Prata, J.C., da Costa, J.P., Duarte, A.C., Rocha-Santos, T. (2019): Methods for sampling and detection of microplastics in water and sediment: A critical review. Trac-Trend Anal Chem 110:150-159. https://doi.org/10.1016/j. trac.2018.10.029

Schwarzböck, T., Aschenbrenner, P., Spacek, S. Szidat, S., Rechberger, H., Fellner, J. (2018): An alternative method to determine the share of fossil carbon in solid refuse-derived fuels-Validation and comparison with three standardized methods. Fuel 220:916-930. https://doi.org/10.1016/ j.fuel.2017.12.076

Schwarzböck, T., Eygen, E., Rechberger, H. Fellner, J. (2016): Determining the amount of waste plastics in the feed of Austrian waste-toenergy facilities. Waste Manage Res 35. https:// doi.org/10.1177/0734242X16660372

Hinweis des Verlags Der Verlag bleibt in Hinblick auf geografische Zuordnungen und Gebietsbezeichnungen in veröffentlichten Karten und Institutsadressen neutral. 